\title{
Fator de desacoplamento em um canavial irrigado no Submédio do Vale do São Francisco ${ }^{1}$
}

\author{
Thieres G. F. da Silva ${ }^{2}$, Sérgio Zolnier ${ }^{3}$, Magna S. B. de Moura ${ }^{4}$, \\ José F. A. do $\mathrm{Carmo}^{4}$ \& Aristides Ribeiro ${ }^{3}$
}

\begin{abstract}
RESUMO
Objetivou-se, com este trabalho, analisar a interação da cana-de-açúcar irrigada com a atmosfera circunvizinha, por meio do fator de desacoplamento $(\Omega)$, para cinco períodos distintos de crescimento e entre eventos de irrigação. O estudo foi conduzido na região do Submédio do Vale do São Francisco. Os dados utilizados para a estimativa de $\Omega$ foram obtidos de sensores acomodados em uma torre micrometeorológica, instalada numa área experimental de cana-de-açúcar, no ciclo de cana-soca. Observou-se que no início do ciclo da cultura (70 a 95 dias após o corte, DAC), o valor médio de $\Omega$ foi 0,74 em decorrência de baixos valores da altura das plantas e do índice de área foliar, devido aos altos valores de velocidade do vento, que reduziram a interação entre a cana-de-açúcar e o ar circunvizinho. Contudo, valor similar foi verificado no período de 309 a 338 DAC, que ocorreu em parte da estação chuvosa, em virtude da evaporação da água depositada sobre o dossel vegetativo. Tanto o tombamento da cana-de-açúcar, ocorrido no período de 218 e 248 DAC, quanto à suspensão da irrigação, ao final do ciclo (339 a 378 DAC) promoveram redução nos valores médios de $\Omega(0,61$ e 0,69 , respectivamente).
\end{abstract}

Palavras-chave: evapotranspiração, interação planta-atmosfera, semiárido

\section{Decoupling factor in an irrigated sugarcane crop in the Lower-Middle Sao Francisco}

\begin{abstract}
The objective of this study was to analyze the interaction between the sugarcane with the surrounding atmosphere, by means of the decoupling factor $(\Omega)$, for five different growth periods and among irrigation events. The study was carried out in an experimental area, located in the region of Lower Middle part of the São Francisco Valley of Brazil. The data used for estimating of $\Omega$ were obtained from sensors accommodated on a micrometeorological tower, which was installed inside the experimental area cultivated with sugarcane ratoon crop It was observed that, at the beginning of the crop cycle (70 to 95 days after cutting, DAC), the average value of $\Omega$ parameter was 0.74 , due to reduced values of plant height and leaf area index, as well as due to the high values of wind speed that reduced the interaction between the sugarcane and the adjacent air. A similar mean value was verified in the period from 309 to 338 DAC, which occurred during the rainy season, due to the evaporation of the water deposited on the canopy. The lodging of the sugarcane plants, which was noticed in the period from 218 and 248 DAC, as well as irrigation suspension at the end of the cycle (339 to $378 \mathrm{DAC}$ ), promoted reduction in the mean values of $\Omega(0.61$ and 0.69 , respectively).
\end{abstract}

Key words: evapotranspiration, plant-atmosphere interaction, Saccharum spp., semiarid 


\section{INTRODUÇÃO}

A cana-de-açúcar é uma planta do tipo $\mathrm{C}_{4}$ que possui rendimentos expressivos sob condições irrigadas, apresentando alta demanda hídrica e eficiência do uso de água ao longo do ciclo de cultivo (Wiedenfeld, 2004; Silva et al., 2011a; 2012). O aumento da necessidade de água em atividades não-agrícolas (abastecimento urbano e animal, industriais, entre outras), incremento dos custos de energia e tarifação da sua utilização, a exigência em prol do aprimoramento da eficiência de irrigação em áreas agrícolas tornou-se urgente, sobremaneira mantendo-se níveis de produção elevados. O uso eficiente de água em sistemas de produção agrícolas pode ser melhorado com base no conhecimento das relações hídricas entre a cultura e o ambiente o que, em particular, permite conhecer os fatores que inflenciam a troca de vapor d'água com a atmosfera (Meinzer \& Grantz, 1989; Inman-Bamber \& Smith, 2005).

A transferência de vapor d'água da superfície vegetada para o ar circunvizinho é um dos processos de maior importância dentre aqueles que ocorrem no balanço de energia à superfície (Silva et al., 2011b). Além do mais, é um processo que atua em conjunto com a fotossíntese e a produtividade primária das plantas (Hao et al., 2007), influenciado pelas condições climáticas e características da superfície (Baldocchi et al., 2004), as quais se manifestam sobre a resistência aerodinâmica $\left(r_{a}\right)$ e da superfície $\left(\mathrm{r}_{\mathrm{s}}\right.$ ). Esta última depende das respostas das plantas e do solo ao ambiente (Kumagai et al., 2004; Nicolás et al., 2008).

Para se avaliar a habilidade das superfícies vegetadas no processo de transferência de vapor d'água para a atmosfera, tem-se utilizado um índice denominado fator de desacoplamento $(\Omega)$ (Marin \& Angelocci, 2011; Takagi et al., 2009). Este indicativo foi proposto, inicialmente, por McNaughton \& Jarvis (1983) e Jarvis \& McNaughton (1986), visto que expressa a conversão de calor sensível da superfície em calor latente, permitindo indicar os fatores que estão governando a evapotranspiração.

O $\Omega$ é baseado na equação de Penman-Monteith, em que seus valores estão compreendidos entre 0 e 1 . No limite inferior, as propriedades atmosféricas, vento e umidade do ar, exercem maior controle sobre a transferência de vapor d'água para atmosfera, haja vista que a evapotranspiração ocorrerá apenas se houver alto déficit de pressão de vapor e baixa resistência aerodinâmica, caso em que se diz que a superfície vegetada está acoplada com a atmosfera. Por outro lado, sob condições em que o fluxo de vapor d'agua é inibido devido à saturação do ar, a maior contribuição ao processo de evapotranspiração se deve à entrada de energia na forma de radiação, caracterizando a extremidade superior do $\Omega$; diz-se, então, que a superfície está desacoplada com a atmosfera. Ambos os casos representam condições extremas de modo que, na realidade, são verificadas situações intermediárias (McNaughton \& Jarvis, 1983; Jones, 1992; DaMatta \& Ramalho, 2006; Goldberg $\&$ Bernhofer, 2008). O $\Omega$ também depende das caracterísiticas da superfície vegetada, de maneira que, quanto maior for sua rugosidade mais fortemente ela interage com a atmosfera, reduzindo a resistência aerodinâmica e aumentando a energia utilizada no processo de evapotranspiração (Steduto \& Hsiao, 1998; Pereira, 2004).

A magnitude de $\Omega$ varia com a disponibilidade de água e os estádios de desenvolvimento das plantas. Sob condições de floresta, onde a disponibilidade de água no solo é reduzida, os valores de $\Omega$ tendem a ser menores que os de culturas agrícolas, evento devido principalmente ao controle estomático das plantas, que inibe a troca de vapor d'água. Adicionalmente, deve-se ao fato da estatura, ao fechamento do dossel e à dimensão das folhas das culturas agrícolas, reduzir a interação do ar com a superfície vegetada. Quando as plantas atingem a fase de senescência os valores de $\Omega$ também aumentam em virtude da diminuição da área foliar transpirante das culturas (Steduto \& Hsiao, 1998).

Com isto, o objetivo deste trabalho foi analisar a variação diurna do fator de desacoplamento para diferentes períodos de crescimento e entre eventos de irrigação em um cultivo de cana-de-açúcar no Submédio do Vale do São Francisco.

\section{Material e mÉtodos}

Este estudo foi conduzido em um talhão experimental com 12,17 ha de cana-de-açúcar (Saccharum spp.), localizado no município de Juazeiro, BA, no Submédio do Vale do São Francisco, que pertence à região semiárida do Nordeste brasileiro. As coordenadas geográficas do local de realização do estudo são: 9० 28' 07" S; 40²2' 43'” O; 386,5 m. A cana-deaçúcar, variedade RB92579, foi conduzida no ciclo de canasoca (segundo ciclo da cultura) na safra de 2007/2008, com plantio ocorrido em fevereiro de 2006 e o primeiro corte em junho de 2007.

O cultivo foi realizado na direção leste-oeste, utilizando-se fileiras com espaçamento de 1,5 m, em solo pertencente à classe dos Vertissolos. A adubação foi feita com base nas análises de solo e foliar aplicando-se $157,5 \mathrm{~kg} \mathrm{ha}^{-1}$ e $0,5 \mathrm{~L} \mathrm{ha}^{-1}$ de ureia e Ajipower, respectivamente. Para o controle de plantas daninhas foi aplicado $1 \mathrm{~L} \mathrm{ha}^{-1}$ dos herbicidas Aminol e Trop. A irrigação era do tipo superficial por sulcos, utilizando-se um sistema de condução em tubos janelados, cujos eventos foram realizados com base no conteúdo de água no solo obtido por meio de uma sonda FDR (Frequency Domain Reflectometer) modelo PR2/6 (Delta-T Devices Ltd., Burwell, Cambridge, UK) e a partir dos valores de evapotranspiração de referência (ETo). Realizouse a irrigação sempre que o conteúdo de água no solo atingia $50 \%$ da capacidade de campo.

Para estimar a evapotranspiração de referência $\left(E T o, \mathrm{~mm} \mathrm{~d}^{-1}\right.$ ) e monitorar as variáveis meteorológicas, foram utilizados os dados de uma estação meteorológica automática (modelo Davis Vantage Pro2, Hayward, CA, USA) instalada em uma área gramada de $20 \mathrm{~m}$ x $20 \mathrm{~m}$, pertencente à Empresa AGROVALE e situada a $3 \mathrm{~km}$ de distância da área experimental. $\mathrm{O}$ método de Penman-Monteith padronizado pela FAO foi utilizado para o cálculo da ETo (Allen et al., 1998):

As variáveis micrometeorológicas (temperatura, velocidade do vento, radiação solar global e fotossinteticamente ativa, saldo de radiação, fluxo de calor no solo) foram medidas por meio de sensores instalados em uma torre com oito metros de 
altura. Esta torre foi posicionada a $350 \mathrm{~m}$ em relação à bordadura, na direção do vento predominante, visando à medição de componentes dos balanços de radiação e de energia.

Utilizaram-se, para o balanço de energia, dois psicrômetros ventilados, sendo os sensores constituídos de termopares do tipo T de Cobre-Constantan, para medida das temperaturas do bulbo seco e do bulbo úmido. Estes psicrômetros foram construídos seguindo os mesmos princípios técnicos informados por Marin et al. (2001) e têm sido utilizados em outros estudos micrometerorológicos realizados na mesma região do presente estudo (Soares et al., 2007; Teixeira et al., 2008). O primeiro psicrômetro foi posicionado a uma altura de $2 \mathrm{z}_{\mathrm{om}}$ do topo do dossel da cultura (em que, $\mathrm{z}_{\mathrm{om}}=$ comprimento de rugosidade da superfície para o momentum), visando garantir seu posicionamento acima da camada rugosa em conformidade, inicialmente, com os valores de $\mathrm{z}_{\mathrm{om}}$ para canade-açúcar, como citados por Cabral et al. (2003). O segundo psicrômetro estava a 1,5 m acima do primeiro, conforme sugerido por Inman-Bamber \& McGlinchey (2003), ou seja, a uma altura de $2 \mathrm{z}_{\mathrm{om}}+1,5 \mathrm{~m}$ em relação ao dossel. Para determinação correta dos gradientes de temperatura e de pressão de vapor d'água, os psicrômetros foram reabastecidos a cada 3 dias, enquanto a troca da gaze do bulbo úmido foi realizada sempre que se verificava a deposição de sujeira e inconsistência física dos dados dos fluxos. Finalmente, em intervalos de duas a três semanas, os psicrômetros foram invertidos entre suas posições de modo que o primeiro foi posicionado no local do segundo, e vice-versa, visando verificar a existência de erros sistemáticos por deficiência de operação dos sensores (Righi et al., 2007).

Quatro conjuntos compostos por sensores de velocidade do vento (03001-5, R.M. Young, Wind Sentry, Campbell Scientific, Logan, Utah, USA), temperatura e umidade relativa do ar (CS215, Campbell Scientific, Logan, Utah, USA) foram instalados também na torre com o objetivo de quantificar as características aerodinâmicas da cana-de-açúcar; dois desses conjuntos foram acomodados na mesma altura dos psicrômetros, e os outros dois em alturas equidistantes; assim, a partir das posições definidas para os psicrômetros os conjuntos de sensores de velocidade do vento, temperatura e umidade relativa do ar foram acomodados logaritmicamente nas seguintes alturas: $2 \mathrm{z}_{\mathrm{om}}, 2 \mathrm{z}_{\mathrm{om}}+0,50 \mathrm{~m}, 2 \mathrm{z}_{\mathrm{om}}+1,50 \mathrm{~m}$ e $2 \mathrm{z}_{\mathrm{om}}$ $+3,50 \mathrm{~m}$. Um saldo radiômetro (NR-Lite Net radiometer, Campbell Scientific, Inc, Logan, Utah) foi instalado na altura de $2 \mathrm{z}_{\mathrm{om}}+1,5 \mathrm{~m}$ (em que $\mathrm{z}_{\mathrm{om}}=$ comprimento de rugosidade da superfície para o momentum), na mesma altura do segundo psicrômetro. Embora as distâncias entre os psicrômetros, anemômetros e do saldo de radiação em relação ao dossel vegetativo tenham sido mantidas até o final do experimento, os mesmos foram deslocados para novos níveis, à medida em que a cultura foi crescendo, conforme realizado por InmanBamber \& Mcglinchey (2003).

No topo da torre foram dispostos sensores de radiação solar global incidente (LI-200SA Quantum sensor, Li-cor, Nebraska, USA) e de radiação fotossinteticamente ativa (LI-190SA Quantum sensor, Li-cor, Nebraska, USA). Três fluxímetros (HFT3-REBS, Campbell Scientific, Inc, Logan, Utah, USA) foram instalados no solo a $0,02 \mathrm{~m}$ de profundidade, entre fileiras e na fileira de cultivo.
Todos os sensores foram conectados a um multiplexador (modelo AM16/32, Campbell Scientific Inc., Logan, Utah, USA) e a um sistema de aquisição de dados (modelo CR10X, Campbell Scientific Inc., Logan, Utah, USA), que foi programado para realizar medições a cada 60 segundos e armazenar médias em intervalos de $15 \mathrm{~min}$. O monitoramento micrometeorológico compreendeu o período de 19/06/2007 (22 DAC) a 09/06/2008 (378 DAC), totalizando 357 dias.

As medidas micrometeorológicas foram utilizadas para a estimativa de $\Omega$, por meio da Eq. 1 (McNaughton \& Jarvis, 1983):

$$
\Omega=\left(1+\frac{\gamma}{\gamma+\Delta} \frac{\mathrm{r}_{\mathrm{s}}}{\mathrm{r}_{\mathrm{a}}}\right)
$$

em que:

$\gamma$ - constante psicrométrica, $\mathrm{kPa}^{\circ} \mathrm{C}^{-1}$

$\Delta$ - tangente da curva de pressão de saturação de vapor d'água, $\mathrm{kPa}^{\circ} \mathrm{C}^{-1}$

$r_{a}$ e $r_{s}$ - resistências aerodinâmica e da superfície $\left(\mathrm{s} \mathrm{m}^{-1}\right)$, respectivamente

Os valores de $\mathrm{r}_{\mathrm{s}}$ foram determinados a partir da inversão da equação de Penman-Monteith (Eq. 2), obtendo-se (Monteith \& Unsworth, 1990):

$$
\mathrm{r}_{\mathrm{s}}=\mathrm{r}_{\mathrm{a}}\left(\frac{\Delta}{\gamma} \frac{\mathrm{H}}{\mathrm{LE}}-1\right)+\left(\frac{\rho_{\mathrm{a}} \mathrm{c}_{\mathrm{p}}\left(\mathrm{e}_{\mathrm{s}}-\mathrm{e}_{\mathrm{a}}\right)}{\gamma \mathrm{LE}}\right)
$$

em que:

$$
\begin{aligned}
& \mathrm{H} \text { - fluxo de calor sensível, } \mathrm{W} \mathrm{m}^{-2} \\
& \mathrm{LE} \text { - fluxo de calor latente, } \mathrm{W} \mathrm{m}^{-2} \\
& \rho_{\mathrm{a}} \text { - densidade do ar, } \mathrm{kg} \mathrm{m}^{-3} \\
& \mathrm{c}_{\mathrm{p}} \text { - calor específico do ar a pressão constante, } \mathrm{J} \mathrm{kg}^{-1}{ }^{\circ} \mathrm{C}^{-1} \\
& \mathrm{e}_{\mathrm{s}}-\mathrm{e}_{\mathrm{a}} \text { - déficit de pressão de vapor d'água, } \mathrm{kPa}
\end{aligned}
$$

Obtiveram-se os valores de LE e de $\mathrm{H}$ através do método do balanço de energia, com base na razão de Bowen (BERB) e analisados em relação às suas consistências físicas, conforme detalhes verificados em Silva et al. (2011a).

O termo $r_{\mathrm{a}}$ foi obtido com a Eq. 3 (Monteith \& Unsworth, 1990):

$$
r_{a}=\frac{\ln \left(\frac{\mathrm{z}-\mathrm{d}}{\mathrm{z}_{\mathrm{oh}}}\right)-\psi_{\mathrm{h}}}{\mathrm{ku}_{*}}
$$

em que:

z - altura de medição dos valores de velocidade do vento acima do dossel da cultura, $m$

d - deslocamento do plano zero, $\mathrm{m}$

$\mathrm{z}_{\text {oh }}$ - comprimento de rugosidade que governa a transferência de calor entre a superfície e a atmosfera, $\mathrm{m}$

k - constante de von $\operatorname{Karman}(0,41)$

$\mathrm{u}_{*}$ - velocidade de fricção, $\mathrm{m} \mathrm{s}^{-1}$

$\psi_{\mathrm{h}}$ - fator de correção da estabilidade atmosférica para transferência de calor sensível, adimensional 
O deslocamento do plano zero (d) foi estimado pelo método estatístico a partir dos valores médios de velocidade do vento para períodos de $30 \mathrm{~min}$, utilizando-se os dados obtidos em condições próximas às da neutralidade (Arya, 2001). Em suma, o método estatístico consiste em utilizar um algoritmo iterativo para maximizar o coeficiente de determinação entre $\ln (\mathrm{z}-\mathrm{d})$ e "u" (Steduto \& Hsiao, 1998; Lyra \& Pereira, 2007). Devido à inércia dos anemômetros de concha, foram desconsiderados os perfis em que a velocidade do vento foi inferior a $1,0 \mathrm{~m} \mathrm{~s}^{-1}$ (Lyra \& Pereira, 2007). O número de Richardson (Ri) foi utilizado para caracterizar as condições de estabilidade atmosférica e, posteriormente, identificar os perfis próximos à neutralidade $(-0,01<\mathrm{Ri}<0,01)$ (Arya, 2001).

Para o cálculo de $\mathrm{u}_{*}$, foi utilizada a relação fluxo-gradiente com dois níveis (1 e 3) de velocidade do vento acima do dossel da cultura, Eq. 4 (Monteith \& Unsworth, 1990):

$$
\mathrm{u}_{*}=\frac{\mathrm{k}\left(\mathrm{u}_{2}-\mathrm{u}_{1}\right)}{\ln \left(\frac{\mathrm{z}_{2}-\mathrm{d}}{\mathrm{z}_{1}-\mathrm{d}}\right)-\psi_{\mathrm{m}}\left(\mathrm{z}_{2}\right)+\psi_{\mathrm{m}}\left(\mathrm{z}_{1}\right)}
$$

em que

$\mathrm{u}_{2}$ e $\mathrm{u}_{1}$ - valores de velocidade do vento obtidos nas alturas $2 \mathrm{z}_{\mathrm{om}}+1,50$ e $2 \mathrm{z}_{\mathrm{om}}$, respectivamente

$\psi_{\mathrm{m}}$ - fator de correção da estabilidade atmosférica para o momentum (adimensional). Os dados de $\psi_{\mathrm{h}}$ e $\psi_{\mathrm{m}}$ foram obtidos conforme citado por Arya (2001)

Obtiveram-se os valores de $\mathrm{z}_{\text {oh }}$ a partir das estimativas de $\mathrm{z}_{\mathrm{om}}$ (parâmetro de rugosidade para o momentum), considerandose a seguinte relação: $\mathrm{z}_{\mathrm{oh}}=0,1 \mathrm{z}_{\mathrm{om}}$ (Allen et al., 1998). Por sua vez, o $z_{\text {om }}$ foi obtido ao longo do ciclo por meio da Eq. 5 (Monteith \& Unsworth, 1990):

$$
\mathrm{z}_{\mathrm{om}}=\frac{\mathrm{z}-\mathrm{d}}{\exp \left(\frac{\mathrm{ku}(\mathrm{z})}{\mathrm{u}_{*}}+\psi_{\mathrm{m}}\right)}
$$

Para avaliar a variação média horária da interação da canade-açúcar com a atmosfera, calculou-se o parâmetro $\Omega$ para 5 períodos representativos do crescimento da cana-de-açúcar (70 a 95 DAC, agosto/2007, fase de estabelecimento e perfilhamento; 126 a $156 \mathrm{DAC}$, outubro/2007, fase de crescimento máximo; 218 a 248 DAC, janeiro/2008, fase de crescimento máximo; 309 a 338 DAC, abril/2008, fase de crescimento máximo e 339 a 378 DAC, maio-junho/2008, fase de maturação), baseados pela altura da cultura $\left(h_{c}\right)$, monitorada ao longo do ciclo por meio de uma trena e do índice de área foliar (IAF), obtido utilizando-se um integrador de área foliar (LAI 2000, LI-COR Inc., Lincoln, NE). Adicionalmente, foram escolhidos dias específicos com base na similaridade das condições meteorológicas e distintos intervalos em relação ao último evento de irrigação, visando analisar a contribuição relativa da resistência da superfície e da energia disponível no processo de troca de vapor d'água entre a cana-de-açúcar e a atmosfera, em escala horária. Os valores do $\Omega$ foram determinados para os horários em que a energia disponível era positiva ( $\mathrm{Rn}-\mathrm{G}>0)$, logo que a maioria dos valores dos fluxos de LE e de $\mathrm{H}$ do período noturno, estimados pelo método BERB, apresentou inconsistência física. Detalhes sobre a análise de qualidade dos dados são mencionados em Silva et al. (2011b).

\section{RESULTADOS E DISCUSSÃO}

No início do ciclo da cana-de-açúcar, que compreendeu o mês de agosto/2007, o intervalo de irrigação foi maior (em média, a cada duas semanas), devido o IAF e a demanda atmosférica ainda serem baixos (Figura 1A, 1B). Um aumento na frequência da irrigação foi evidenciado a partir do mês de outubro/2007, quando os valores de ETo atingem valores máximos (Figura 1B). Constata-se que, entre os meses de março e abril/2008, o número de irrigações foi reduzido em razão da ocorrência dos eventos de precipitação pluviométrica, a qual respondeu por $48 \%$ do volume total de chuva ao longo do ciclo da cultura. A partir do mês de maio/2008, os eventos de chuva foram reduzidos e as irrigações suspensas para promover a maturação da cultura e propiciar a colheita mecanizada, por se tratar de um solo da classe dos vertissolos.

Com base nos dados do perfil logarítmico do vento, verificou-se que o valor do deslocamento do plano zero em relação à altura do dossel da cultura $\left(\mathrm{d} / \mathrm{h}_{\mathrm{c}}\right)$ foi igual a 0,71 ,

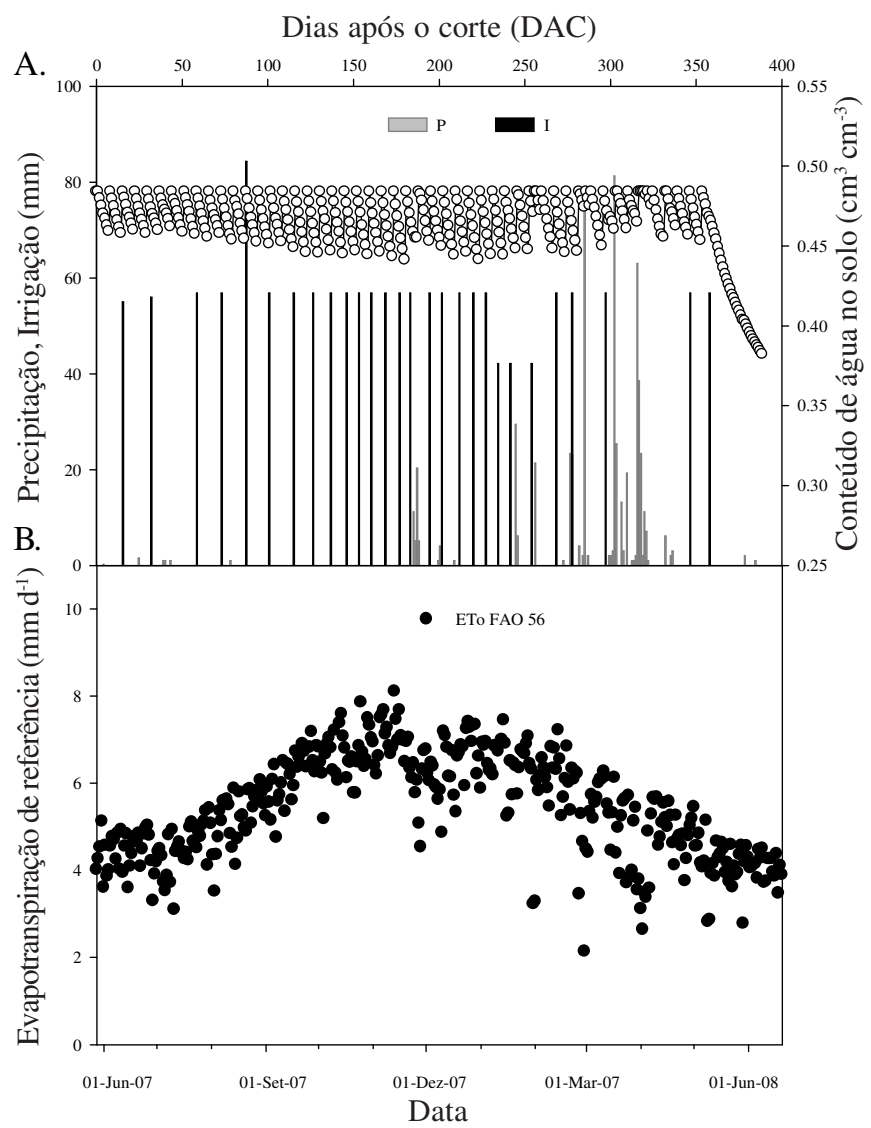

Figura 1. Dados de precipitação e irrigação (A) e de evapotranspiração de referência (B) ao longo do ciclo da cana-de-açúcar variedade RB92579 irrigada (cana-soca), sob as condições climáticas do Submédio do Vale do São Francisco 
enquanto a relação entre o parâmetro de rugosidade, estimado ao longo do ciclo, com a altura do dossel da cultura $\left(\mathrm{z}_{\mathrm{om}} / \mathrm{hc}\right)$ resultou em valores médios de $0,09 \mathrm{~m}$, aproximadamente. Tais resultados foram semelhantes aos informados por Cabral et al. (2003) e são consistentes com aqueles informados por Monteith \& Unsworth (1990), que citam valores de $\mathrm{d} / \mathrm{h}_{\mathrm{c}}$ entre 0,6 e $0,7 \mathrm{e}$ de $\mathrm{z}_{\text {om }} /$ hc entre 0,08 e 0,12 .

De modo geral, observou-se, ao amanhecer e ao entardecer, que os valores de $\Omega$ foram menores do que para outros períodos do dia $(\Omega<0,4)$, indicando um acoplamento maior da cultura com a atmosfera (Figura 2) tendência que se deve aos altos valores de $r_{s}$ em virtude do fechamento dos estômatos que, por sua vez, ocorreu como resultado da baixa disponibilidade de radiação (Figura 3).

De acordo com a Tabela 1 , o valor médio diário de $\Omega$ foi 0,74 no início do ciclo (70 - 95 DAC, agosto/2007) quando a canade-açúcar possuía IAF em torno de $0,9 \pm 0,1 \mathrm{~m}^{2} \mathrm{~m}^{-2}$ e $\mathrm{h}_{\mathrm{c}}$ de 1,0 $\mathrm{m}$. Neste período, a radiação solar global incidente medida foi $16,3 \mathrm{MJ} \mathrm{m}^{-2} \mathrm{~d}^{-1}$. O valor do parâmetro $\Omega$ mostra a existência de uma pequena interação do dossel vegetativo com a atmosfera, sinalizando que o desacoplamento era moderado a alto uma vez que culturas agrícolas bem irrigadas apresentam alto desacoplamento com valores de $\Omega$ superiores a 0,8 (Jarvis \& McNaughton, 1986; Meinzer \& Grantz, 1989) e espécies florestais possuem baixo desacoplamento, com valores de $\Omega$ inferiores a 0,2 (Jarvis \& McNaughton, 1986). No início do ciclo das culturas, quando se tem IAF e h pequenos, caracterizando o baixo dossel vegetativo, comumente os valores diários de $\Omega$ são altos, indicando efeitos menos pronunciados do DPV e da $r_{a}$ sobre a troca de vapor d'água entre a cultura e a atmosfera (Jones, 1992; Steduto \& Hsiao, 1998).

Em escala horária observou-se que, devido à redução da $r_{s}$, o parâmetro $\Omega$ variou rapidamente no início do período diurno até alcançar valores situados entre 0,50 e 0,60 por volta das $8 \mathrm{~h}$ (Figura 2A). Neste período a quantidade de energia incidente, em termos de radiação fotossinteticamente ativa (RFA), era inferior a $600 \mu \mathrm{mol} \mathrm{m}^{-2} \mathrm{~s}^{-1}$ (Figura 3A). Constatou-se, então, um acréscimo menos expressivo do desacoplamento a partir das $8 \mathrm{~h}$, atingindo valores em torno de 0,70 $\pm 0,02$ entre $11 \mathrm{e} 14 \mathrm{~h}$, quando a RFA atingiu valores em torno de $2000 \mu \mathrm{mol} \mathrm{m}^{-2} \mathrm{~s}^{-1}$. O aumento de $\Omega$ esteve associado à maior disponibilidade de energia para a troca de vapor d'água com a atmosfera, representada pelo fluxo de calor latente (LE) (Figura 3A), que indicou valores em torno de $456 \pm 25 \mathrm{~W} \mathrm{~m}^{-2}$. Neste período, verificou-se, entre 11 e 14 h, que o parâmetro $\Omega$ aumentou apesar do incremento da velocidade do vento $(\mathrm{u})\left(3,8 \pm 0,4 \mathrm{~m} \mathrm{~s}^{-1}\right)$, devido aos altos valores de $\mathrm{u}$ induzir o fechamento estomático das plantas (El-Sharkawy et al., 1990).

Steduto \& Hsiao (1998) verificaram, para a cultura do milho (IAF $\sim 5,5 \mathrm{~m}^{2} \mathrm{~m}^{-2} \mathrm{e} \mathrm{h}_{\mathrm{c}} \sim 3,10 \mathrm{~m}$ ) em torno do meio-dia, que os valores de $\Omega$ tenderam a aumentar $(>0,40)$, quando a velocidade do vento apresentou valores entre 4 a $5 \mathrm{~m} \mathrm{~s}^{-1}$. Porém, no início do ciclo da cultura (IAF de $0,58 \mathrm{~m}^{2} \mathrm{~m}^{-2}$ e $\mathrm{h}_{\mathrm{c}}$ de $30 \mathrm{~cm}$ ), um aumento de $\Omega$ também foi verificado (entre 0,45 e 0,65 ) quando os valores de velocidade do vento foram menores que $1,5 \mathrm{~m} \mathrm{~s}^{-1}$, em virtude de reduzir a interação do ar circunvizinho com a superfície vegetada. Valores situados entre 1,5 e $4 \mathrm{~m} \mathrm{~s}^{-1}$ induzem,

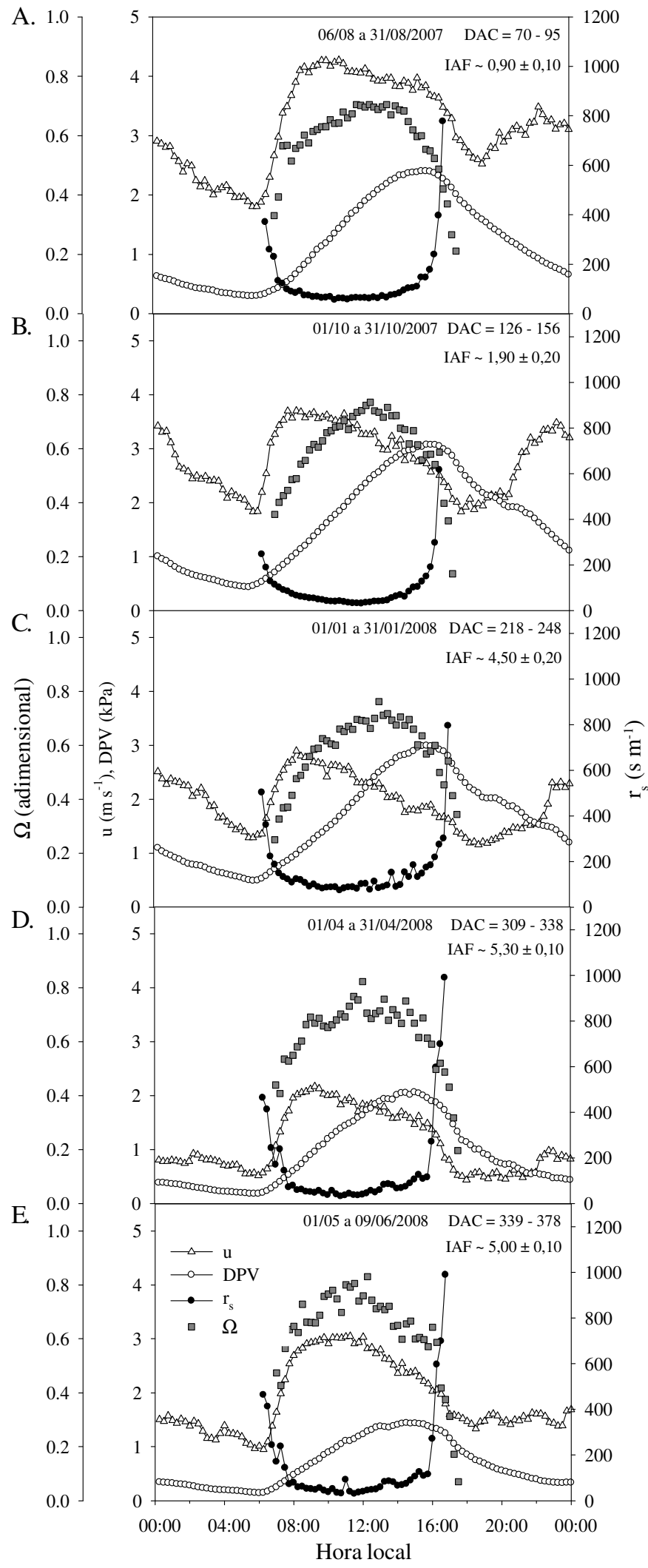

Figura 2. Variação média horária do fator de desacoplamento $(\Omega)$, velocidade do vento $(u)$, déficit de pressão de saturação de vapor d'água (DPV) e resistência da cultura $\left(r_{s}\right)$ para 5 períodos representativos do crescimento da cana-de-açúcar variedade RB92579 irrigada (cana-soca), sob as condições climáticas do Submédio do Vale do São Francisco 


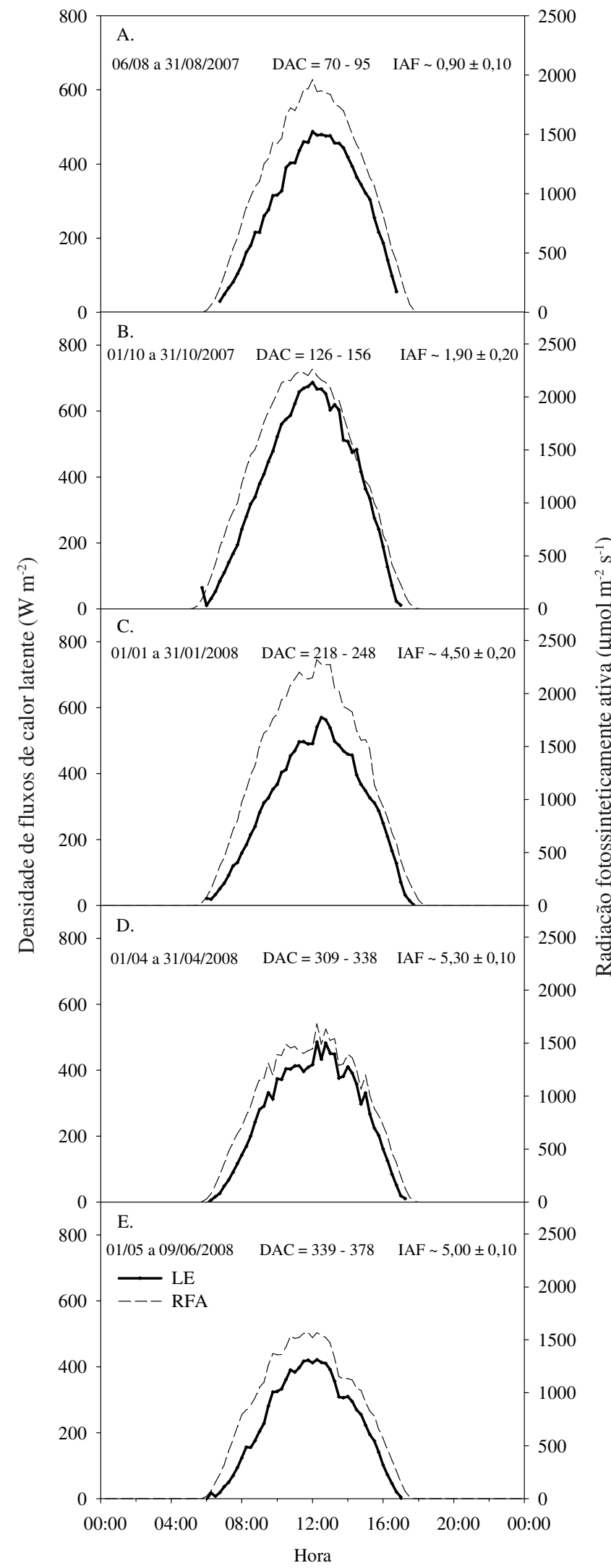

Figura 3. Variação média horária da densidade do fluxo de calor latente (LE) e da radiação fotossinteticamente ativa (RFA) para 5 períodos representativos do crescimento da cana-de-açúcar variedade RB92579 irrigada (canasoca), sob as condições climáticas do Submédio do Vale do São Francisco provavelmente, a uma interação maior entre a superfície da cultura e a atmosfera, como resultado do maior contato do dossel com o ar circunvizinho. Resultados diferentes foram encontrados por Pereira (2004), que observou uma correlação negativa entre a velocidade do vento e os valores diários de $\Omega$ para a grama. Tais informações permitem inferir que numa superfície gramada, em que o dossel é mais fechado, o aumento da velocidade do vento permite melhorar sua interação com a atmosfera. Por outro lado, em culturas com portes maiores, como o caso do milho e da cana-de-açúcar, os altos valores de velocidade do vento podem inibir esta interação, em razão de afetar o comportamento estomático das plantas.

Observa-se, entre as 14 e $16 \mathrm{~h}$, maior acoplamento da canade-açúcar com a atmosfera, coincidindo com elevados valores de DPV (Figura 2A). Em escala diária o DPV foi, em média, igual a 1,45 kPa, com os maiores valores ocorrendo entre 14 e $18 \mathrm{~h}$; contudo, a influência do DPV foi evidente apenas até as $16 \mathrm{~h}$; a partir deste momento, observou-se que os valores da resistência do dossel da cultura (rs) apresentaram maior importância devido à diminuição da radiação incidente, que foi inferior a $650 \mu \mathrm{mol} \mathrm{m}^{-2} \mathrm{~s}^{-1}$ (Figura 3A). Para o período analisado (agosto/2007), constatou-se que os menores valores de rs ocorreram durante o período da manhã, com ligeiro aumento à tarde. Em termos médios diários, a rs foi de $53 \mathrm{~s} \mathrm{~m}^{-1}$.

No período entre 126 e 156 DAC (outubro/2007) (Figura

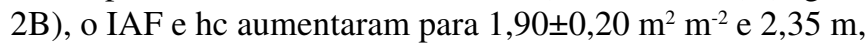
respectivamente. Em comparação ao período anteriormente analisado, a disponibilidade de radiação solar foi superior sendo, em média, igual a 22,8 $\mathrm{MJ} \mathrm{m}^{-2} \mathrm{~d}^{-1}$ (Tabela 1). Por outro lado, verificou-se aumento do DPV, com média diária de 2,07 kPa, enquanto a velocidade do vento foi de $2,55 \mathrm{~m} \mathrm{~s}^{-1}$. Assim, constatou-se redução dos valores de $\Omega(\sim 0,70)$ em relação ao período anterior e, em escala horária, o $\Omega$ aumentou continuamente até próximo do meio-dia $(11-14 \mathrm{~h})$, quando atingiu valores máximos $(\sim 0,73 \pm 0,03)$ (Figura $2 \mathrm{~B}$ ); mesmo assim, neste período os valores de $\Omega$ não diferiram expressivamente do período anterior (Figura 1).

Esta mesma tendência foi evidenciada para os períodos subsequentes, indicando a ocorrência de um desacoplamento máximo no período de maior suprimento de radiação (Figura 3); entre 11 e $14 \mathrm{~h}$ os valores de RFA foram de $2089 \pm$ $178 \mu \mathrm{mol} \mathrm{m}^{-2} \mathrm{~s}^{-1}$, ao passo que os valores de LE atingiram $626 \pm$ $58 \mathrm{~W} \mathrm{~m}^{-2}$; em seguida, verificou-se uma pequena redução de $\Omega$ até próximo das $16 \mathrm{~h}$ devido, sem dúvida, ao aumento dos valores de DPV (> 3,0 kPa). Por outro lado, a velocidade do vento se manteve moderada, estando situada entre 2 e $3 \mathrm{~m} \mathrm{~s}^{-1}$ (Figura 2B), que também pode ter contribuído para o aumento do acoplamento entre a cultura e a atmosfera (Steduto \& Hsiao, 1998). A partir das $16 \mathrm{~h}$ houve rápida redução de $\Omega$ em decorrência da diminuição da disponibilidade de radiação (< $700 \mu \mathrm{mol} \mathrm{m} \mathrm{m}^{-2} \mathrm{~s}^{-1}$ ) (Figura 3B). Quando comparados com o período anterior, verificou-se pouca redução dos valores de $r_{s}$, (aproximadamente $49 \mathrm{~s} \mathrm{~m}^{-1}$ ) além de decréscimo expressivo dos valores de $\mathrm{r}_{\mathrm{a}}\left(\mathrm{de} 46\right.$ para $\left.29 \mathrm{~s} \mathrm{~m}^{-1}\right)$ (Tabela 1$)$.

No período de 218 a 248 DAC (janeiro/2008) (Figura 2C), os valores de $\Omega$ tenderam a ser ligeiramente menores, resultado do tombamento da cana-de-açúcar, fato observado na área experimental que, inicialmente, induz a redução da área foliar 
Tabela 1. Valores médios do fator de desacoplamento $(\Omega)$ e de variáveis correspondentes para 5 períodos representativos do crescimento da cultura da cana-deaçúcar variedade RB92579 irrigada (cana-soca), sob as condições climáticas do Submédio do Vale do São Francisco

\begin{tabular}{lccccc}
\hline \multirow{2}{*}{ Variáveis } & \multicolumn{5}{c}{ Período de crescimento } \\
\cline { 2 - 6 } & Ago/07 & Out/07 & Jan/08 & Abr/08 & Mai-Jun/08 \\
DAC & 70 a 95 & 126 a 156 & 218 a 248 & 309 a 338 & 339 a 378 \\
$\Omega$ & 0,74 & 0,70 & 0,61 & 0,75 & 0,69 \\
IAF & 0,90 & 1,90 & 4,50 & 5,30 & 5,00 \\
$\mathrm{~h}_{\mathrm{c}}$ & 1,00 & 2,35 & 3,95 & 4,32 & 4,35 \\
$\mathrm{DPV}$ & 1,45 & 2,07 & 2,13 & 1,01 & 1,14 \\
$\mathrm{Rg}$ & 16,3 & 22,8 & 22,6 & 17,2 & 15,4 \\
$\mathrm{u}$ & 3,10 & 2,55 & 1,86 & 1,34 & 1,81 \\
$\mathrm{Z}_{\text {om }}$ & 0,08 & 0,10 & 0,27 & 0,17 & 0,51 \\
$\mathrm{r}_{\mathrm{s}}$ & 53 & 49 & 80 & 39 & 50 \\
$\mathrm{r}_{\mathrm{a}}$ & 46 & 29 & 32 & 30 & 27
\end{tabular}

DAC - dias após o corte; IAF - índice de área foliar, $\mathrm{m}^{2} \mathrm{~m}^{-2} ; \mathrm{h}_{\mathrm{c}}$ - altura da cultura, $\mathrm{m}$; DPV - déficit de pressão de saturação de vapor d'água, $\mathrm{kPa}$; $\mathrm{Rg}$ - radiaçấo solar global incidente, $\mathrm{MJ} \mathrm{m}^{-2} \mathrm{~d}^{-1}$; $\mathrm{u}$ - velocidade do vento, $\mathrm{m} \mathrm{s}^{-1} ; \mathrm{z}_{\mathrm{om}}$ - parâmetro de rugosidade da superfície, $\mathrm{m} ; \mathrm{r}_{\mathrm{s}}$ - resistência da superfície do dossel da cultura, $\mathrm{s} \mathrm{m}^{-1} ; \mathrm{r}_{\mathrm{a}}$ - resistência aerodinâmica, $\mathrm{s} \mathrm{m} \mathrm{m}^{-1}$

verde. Neste período, a velocidade do vento foi $1,86 \mathrm{~m} \mathrm{~s}^{-1}$, inferior ao verificado entre os 126 e 156 DAC (outubro/2007), enquanto a Rg não variou expressivamente, sendo igual a 22,64 $\mathrm{MJ} \mathrm{m}^{-2} \mathrm{~d}^{-1}$. Durante este período, a $\mathrm{r}_{\mathrm{s}}$ apresentou valores de 80 $\mathrm{s} \mathrm{m}^{-1}$, Este aumento de $\mathrm{r}_{\mathrm{s}}$ resultou numa redução de LE que, entre 11 e $14 \mathrm{~h}$, foi igual a $506 \pm 36 \mathrm{~W} \mathrm{~m}^{-2}$, mesmo tendo sido constatada uma quantidade maior de radiação para as plantas (RFA de aproximadamente $2133 \pm 148 \mu \mathrm{mol} \mathrm{m} \mathrm{m}^{-2} \mathrm{~s}^{-1}$ ). Adicionalmente, os valores de DPV foram maiores (Figura $2 \mathrm{C} \mathrm{e}$ Tabela 1), o que promoveu o aumento do acoplamento entre a cana-de-açúcar e a atmosfera; já entre 11 e 14 h, o $\Omega$ apresentou valores em torno de $0,69 \pm 0,03$; em escala diária, o valor médio foi igual a 0,61 (Tabela 1 ).

Nos dois períodos seguintes (309 a 338DAC, abril/2008; 339 a 378 DAC, maio-junho/2008), constatou-se um aumento dos valores de $\Omega$, resultante, provavelmente, do retorno dos eventos de irrigação, da menor demanda atmosférica e da redução inexpressiva do IAF ao final do ciclo.

O mês de abril/2008 (Figura 2D) foi caracterizado por estar compreendido na parte final da estação chuvosa, o que favoreceu o aumento de $\Omega$ em virtude, provavelmente, da evaporação da água depositada sobre a superfície vegetada da cana-de-açúcar. Neste período, a $\operatorname{Rg}$ foi de $17,2 \mathrm{MJ} \mathrm{m}^{-2} \mathrm{~d}^{-1} \mathrm{e}$ a velocidade do vento de $1,34 \mathrm{~m} \mathrm{~s}^{-1}$, enquanto o DPV foi de 1,01 $\mathrm{kPa}$. Os valores de $\mathrm{r}_{\mathrm{s}}$ foram, em média, iguais a $39 \mathrm{~s} \mathrm{~m}^{-1}$, indicando o baixo estresse sofrido pela cultura (Meinzer \& Grantz, 1989). Em decorrência do menor controle da cultura, a transferência de vapor d'água para a atmosfera, quando comparada ao período anterior, propiciou maior quantidade de energia destinada ao processo de evapotranspiração. Em escala horária, quando a disponibilidade de radiação fotossinteticamente ativa era de $1464 \pm 113 \mu \mathrm{mol} \mathrm{m}^{-2} \mathrm{~s}^{-1}(11 \mathrm{a} 14 \mathrm{~h})$, o LE foi de $425 \pm 34$ $\mathrm{W} \mathrm{m} \mathrm{m}^{-2}$ enquanto os valores de $\Omega$ estiveram situados em torno de $0,73 \pm 0,04$.

Entre os 339 e 378 DAC (maio-junho/2008) (Figura 2E), verificou-se, devido à suspensão da irrigação, redução dos valores de $\Omega$, sendo em média igual a 0,69 (Tabela 1). É provável que esta redução esteja associada ao próprio tombamento observado da cana-de-açúcar, que acarreta aumento da rugosidade da superfície $\left(\mathrm{z}_{\mathrm{om}}\right)$. Para este período, verificou-se que $z_{\text {om }}$ foi, em média, igual a $0,51 \mathrm{~m}$ (Tabela 1). Resultado semelhante obtiveram Cabral et al. (2003) que confirmam um aumento de $\mathrm{z}_{\text {om }}$ em torno de 0,50 m devido ao tombamento da cultura. Associado a essas informações também se tem que, durante o período final do ciclo da cana-de-açúcar, a velocidade do vento $\left(1,81 \mathrm{~m} \mathrm{~s}^{-1}\right)$, juntamente com o DPV $(1,14 \mathrm{kPa})$, foi superior à do período antecedente; os valores mencionados induziram o aumento da $\mathrm{r}_{\mathrm{s}}\left(\mathrm{em}\right.$ torno de $50 \mathrm{~s} \mathrm{~m}^{-1}$ ) (Tabela 1) e, consequentemente, os valores de $\Omega$. Em termos horários, no intervalo (11 - 14 h) em que o suprimento de radiação era máximo (aproximadamente $1426 \pm 174 \mu \mathrm{mol} \mathrm{m} \mathrm{m}^{-2} \mathrm{~s}^{-1}$ ), os valores de $\Omega$ foram de $0,75 \pm 0,05$, apresentando fluxo de calor latente de 381 $\pm 45 \mathrm{~W} \mathrm{~m}^{-2}$ (Figuras 2E e $3 \mathrm{E}$ ).

Meinzer \& Grantz (1989) citam que, sob condições de estresse hídrico severo, a cana-de-açúcar tende a apresentar valores de $\Omega$ em torno de 0,20 . Assim, apesar da redução dos valores de $\Omega$ no período entre 339 e 378 DAC (maio-junho/2008), verificou-se que o desacoplamento foi caracterizado como sendo moderado (Steduto \& Hsiao, 1998). Tal resultado pode estar relacionado ao fato do IAF não ter apresentado decréscimos expressivos $\left(5,3\right.$ para $\left.5,0 \mathrm{~m}^{2} \mathrm{~m}^{-2}\right)$. Além do mais, a cultura não foi submetida a condições de estresse severo visto que o período máximo de suspensão de irrigação foi inferior a 44 dias, de modo que não foram evidenciadas reduções do acúmulo de sacarose que, normalmente, são observadas em condições de estresse hídrico (Inman-Bamber \& Smith, 2005). Segundo Steduto \& Hsiao (1998), reduções nos valores de $\Omega$ são verificadas apenas quando há restrições severas de água no solo, as quais promovem reduções significativas dos valores de $\operatorname{IAF}\left(5,4\right.$ para $\left.2,1 \mathrm{~m}^{2} \mathrm{~m}^{-2}\right)$, de maneira que a planta tende a controlar a transferência de água por meio do fechamento dos estômatos.

Para analisar a variação diurna dos valores de $\Omega$ em períodos entre eventos de irrigação, realizou-se uma análise para dois dias distintos, que envolveram o mesmo período de crescimento da cultura e com valores similares de radiação solar global, velocidade do vento e déficit de pressão de vapor d'água no ar (Figuras 4A e 4B). O primeiro (12/02/2008) foi caracterizado pela ocorrência de 13 dias após o último evento de irrigação enquanto o segundo (15/02/2008) correspondeu a 2 dias após a última irrigação. Nas Figuras $5 \mathrm{~A}$ e $5 \mathrm{~B}$ são destacados os valores de RFA e LE dos dois dias analisados.

Verifica-se que a variação dos valores de "u" e DPV é bem semelhante, sendo que os maiores valores de "u" ocorreram durante o período da manhã, enquanto os do DPV foram observados nos horários da tarde, com valores máximos semelhantes entre os dois dias analisados. Por outro lado, é possível observar, para o dia 12/02/2008, que os valores de $\Omega$ são bem menores quando comparados aos do dia 15/02/2008. Para o primeiro dia (Figura $4 \mathrm{~A}$, os valores de $\Omega$ foram baixos entre 11 e $14 \mathrm{~h}$ (aproximadamente $0,46 \pm 0,06$ ), caracterizando um forte acoplamento entre a cana-de-açúcar e a atmosfera. Neste período, os valores correspondentes de RFA e LE foram, respectivamente, $2122 \pm 236 \mu \mathrm{mol} \mathrm{m}^{-2} \mathrm{~s}^{-1} \mathrm{e} 420 \pm 43 \mathrm{~W} \mathrm{~m}^{-2}$ (Figura $5 \mathrm{~A})$. Após as $14 \mathrm{~h}$, foi possível perceber valores de $\Omega$ ainda menores, indicando o aumento do acoplamento entre a cultura 


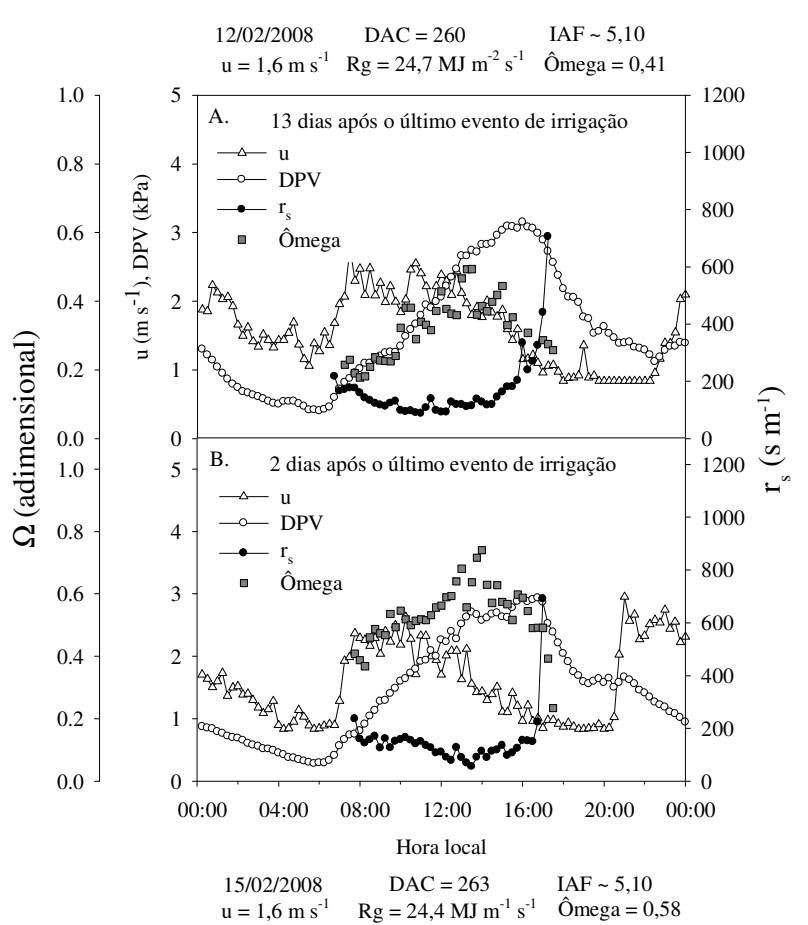

Figura 4. Valores médios horários do fator de desacoplamento (Ômega), velocidade do vento (u) e resistência da superfície $\left(r_{s}\right)$ para dois dias distintos, transcorridos após o último evento de irrigação em um cultivo de cana-deaçúcar variedade RB92579 irrigada (cana-soca), sob as condições climáticas do Submédio do Vale do São Francisco

A.

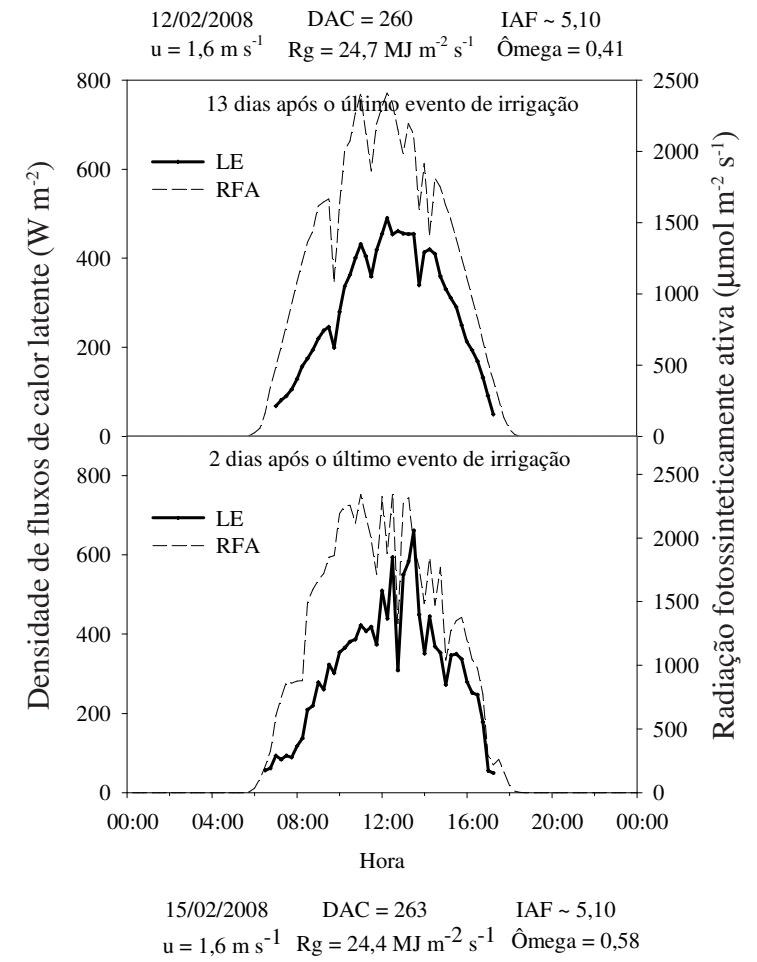

Figura 5. Valores médios horários da densidade de fluxos de calor latente (LE) e de radiação fotossinteticamente ativa (RFA) para dois dias distintos, transcorridos após o último evento de irrigação em um cultivo de cana-de-açúcar variedade RB92579 irrigada (cana-soca), sob as condições climáticas do Submédio do Vale do São Francisco e a atmosfera em decorrência da elevação dos valores de $r_{s}$, que, por sua vez, são devidos aos maiores valores de DPV. Considerando-se o período diurno, os valores de $\mathrm{r}_{\mathrm{s}}$ foram, em média, iguais a $103 \mathrm{~s} \mathrm{~m}^{-1}$, ao passo que os de $\Omega$ se situaram em torno de 0,41 , resultados que indicam controle expressivo da cultura ao processo de troca de vapor d'água com a atmosfera, ou seja, um acoplamento significativo (Steduto \& Hsiao, 1998).

Por outro lado, quando se analisou o dia 15/02/2008 (Figura 4B), foi possível verificar menor acoplamento da cana-de-açúcar com a atmosfera. Considerando-se o período diurno, o valor de $\Omega$ foi, em média, igual a 0,58 , caracterizando um acoplamento moderado enquanto a $\mathrm{r}_{\mathrm{s}}$ média foi de $93 \mathrm{~s} \mathrm{~m}^{-1}$. Durante este dia, o $\Omega$ aumentou rapidamente após as $8 \mathrm{~h}$, mesmo com o aumento da $r_{\mathrm{s}}$ em razão dos valores de DPV mais acentuados. Os valores máximos de $\Omega$ ocorreram entre 11 e $14 \mathrm{~h}$, com valores em torno de $0,62 \pm 0,07$, sendo que uma pequena redução nos valores de $r_{s}$ foi verificada neste período. A RFA foi de $2122 \pm$ $236 \mu \mathrm{mol} \mathrm{m}^{-2} \mathrm{~s}^{-1}$ e oLE de $523 \pm 219 \mathrm{~W} \mathrm{~m}^{-2}$ (Figura 5B). Observouse, entre 14 e $16 \mathrm{~h}$, um aumento do acoplamento, por causa do aumento da $r_{s}$, sendo que valores menores de $\Omega$ foram observados ao final da tarde (Figura 4B).

\section{CONCLusões}

1. Com exceção do período inicial do ciclo da cana-de-açúcar (agosto/2007), quando o fator de desacoplamento aumentou, nos demais períodos (outubro/2007, janeiro/2008, abril/2008 e maio-junho/2008) não foram observadas diferenças expressivas nos valores médios horários do acoplamento da cana-de-açúcar com a atmosfera.

2. Os períodos de 70 a 95 DAC (agosto/2007) e de 309 a 338 DAC (abril/2008) foram os que resultaram em maiores valores do fator de desacoplamento.

3. Em termos diários constatou-se que no período compreendido de 218 a 248 DAC (janeiro/2008), caracterizado pelo tombamento da cana-de-açúcar, ocorreu ligeira redução nos valores do fator de desacoplamento; resultado semelhante também foi observado no final do ciclo.

4. Verificou-se que o acoplamento da cana-de-açúcar é baixo nos primeiros dias após os eventos de irrigação, mas aumenta posteriormente, acarretando em consequência do aumento da resistência de superfície da cultura.

\section{AgRAdECIMENTOS}

À Empresa Agroindústrias do Vale do São Francisco S.A. e à Empresa Brasileira de Pesquisa Agropecuária, Embrapa Semiárido, pelas infraestruturas concedidas para execução do presente estudo.

\section{LITERATURA CITADA}

Allen, R. G.; Pereira, L. S.; Raes, D.; Smith, M. Crop evapotranspiration - guidelines for computing crop water requirements. Rome: FAO, 1998. 326 p. FAO. Irrigation and Drainage Paper, 56 
Arya, S. P. Introduction to micrometeorology. San Diego: Academic Press, 2001. 420p.

Baldocchi, D. D.; Xu, L. K.; Nancy, K. How plant functional type, weather, seasonal drought, and soil physical properties alter water and energy fluxes of an oak-grass savanna and an annual grassland. Agricultural and Forest Meteorology, v.123, p.13-39, 2004.

Cabral, O. M. R.; Rocha, H. R.; Ligo, M. A. V.; Brunini, O.; Dias, M.A. F. S. Fluxos turbulentos de calor sensível, vapor d'água e $\mathrm{CO}_{2}$ sobre plantação de cana-de-açúcar (Saccharum sp.) em Sertãozinho-SP. Revista Brasileira de Meteorologia, v.18, p.61-70, 2003.

DaMatta, F. M.; Ramalho, J. D. C. Impacts of drought and temperature stress on coffee physiology and production: a review. Brazilian Journal Physiology, v.18, p.55-81, 2006.

El-Sharkawy, M. A. Effect of humidity and wind on leaf conductance of field grown cassava. Revista Brasileira Fisiologia Vegetal, v.2, p.17-22, 1990.

Goldberg, V.; Bernhofer, C. Testing different decoupling coefficients with measurements and models of contrasting canopies and soil water conditions. Annales Geophysicae, v.26, p.1977-1992, 2008.

Hao, Y.; Wang, Y.; Huang, X.; Cui, X.; Zhou, X.; Wang, S.; Niu, H.; Jiang, G. Seasonal and interannual variation in water vapor and energy exchange over a typical steppe in Inner Mongolia, China. Agricultural and Forest Meteorology, v.146, p.57-69, 2007.

Inman-Bamber, N. G.; Mcglinchey, M. G. Crop coefficients and water-use estimates for sugarcane based on long-term bowen ratio energy balance measurements. Field Crops Research, v.83, p.125-138, 2003.

Inman-Bamber, N. G.; Smith, D. M. Water relations in sugarcane and response to water deficits. Field Crops Research, v.92, p.185-202, 2005.

Jarvis, P. G., McNaughton, K. G. Stomatal control of transpiration: scaling up from leaf to region. Advances Ecology Research, v.15, p.1-49, 1986.

Jones, H. G. Plants and microclimate. Cambridge: University Press Cambridge, 1992.428p.

Kumagai, T.; Saitoh, T. M.; Sato, Y.; Morooka, T.; Manfroi, O. J.; Kuraji, K.; Suzuki, M. Transpiration, canopy conductance and the decoupling coefficient of a lowland mixed dipterocarp forest in Sarawak, Borneo: Dry spell effects. Journal of Hydrology, v.287, p.237-251, 2004.

Lyra, G. B., Pereira, A. R. Dificuldades de estimativa dos parâmetros de rugosidade aerodinâmica pelo Perfil logarítmico do vento sobre vegetação esparsa em região semiárida. Revista Brasileira de Geofísica, v.25, p.187-197, 2007.

Marin, F. R.; Angelocci, L. R. Irrigation requirements and transpiration coupling to the atmosphere of a citrus orchard in Southern Brazil, Agricultural Water Management, v.98, p.1091-1096, 2011.

Marin, F. R.; Angelocci, L. R.; Coelho Filho, M. A.; Nova, N. A. V. Construção e avaliação de psicrômetro aspirado de termopar. Scientia Agricola, v.58, p.839-844, 2001.
McNaughton, K. G.; Jarvis, P. G. Predicting effects of vegetation changes on transpiration and evaporation. In: Kozlowski, T. T. (ed.). Water deficits and plant growth. New York: Academic Press, 1983. v.7, p.1-47.

Meinzer, F. C.; Grantz, D. A. Stomatal control of transpiration from a developing sugarcane canopy. Plant Cell and Environmental, v.12, p.635-642, 1989.

Monteith, J. L.; Unsworth, M. H. Principles of environmental physics. 2.ed. London: Edward Arnold, 1990. 291p.

Nicolás, E.; Barradas, V. L.; Ortuño, M. F.; Navarro,A.; Torrecillas, A.; Alarcón, J. J. Environmental and stomatal control of transpiration, canopy conductance and decoupling coefficient in young lemon trees under shading net. Environmental and Experimental Botany, v.63, p.200-206, 2008.

Pereira, A. R. The Priestley-Taylor parameter and the decoupling factor for estimating reference evapotranspiration. Agricultural and Forest Meteorology, v.125, p.305-313, 2004.

Righi, E. Z.; Angelocci, R. L.; Marin, F. R. Energy balance of a young drip-irrigated coffee crop in southeast Brazil: an analysis of errors and reliability of measurements by the Bowen ratio method. Revista Brasileira de Agrometeorologia, v.15, p.267-279, 2007.

Silva, T. G. F. da; Moura, M. S. B. de; Zolnier, S.; Soares, J. M.; Souza, L. S. B. de; Brandão, E. O. Variação do balanço de radiação e de energia da cana-de-açúcar irrigada no semiárido brasileiro. Revista Brasileira de Engenharia Agrícola e Ambiental, v.15, p.139-147, 2011a.

Silva, T. G. F. da; Moura, M. S. B. de; Zolnier, S.; Soares, J. M.; Vieira, V. J. de S. Farias Júnior, W. G. Demanda hídrica e eficiência do uso de água da cana-de-açúcar irrigada no semiárido brasileiro. Revista Brasileira de Engenharia Agrícola e Ambiental, v.15, p.1257-1265, 2011 b.

Silva, T. G. F. da; Moura, M. S. B. de; Zolnier, S.; Soares, J. M.; Vieira, V. J. de S. Farias Júnior, W. G. Requerimento hídrico e coeficiente de cultura da cana-de-açúcar irrigada no semiárido brasileiro. Revista Brasileira de Engenharia Agrícola e Ambiental, v.16, p.64-71, 2012.

Soares, J. M.; Azevedo, P. V. de; Silva, B. B. da. Bowen ratioenergy balance associated errors in Vineyards under dripping irrigation. Revista Brasileira de Meteorologia, v.22, p.233-240, 2007.

Steduto, P.; Hsiao, T. C. Maize canopies under two soil water regimes. III. Variation in coupling with the atmosphere and role of leaf area index. Agricultural and Forest Meteorology, v.89, p.201-213, 1998.

Takagi, K.; Kimura, R.; Saylan, L. Variability of surface characteristics and energy flux patterns of sunn hemp (Crotalaria juncea L.) under well-watered conditions. Theoretical Applied Climatology, v.96, p.261-273, 2009.

Teixeira, A. H. C.; Bastiaanssen, W. G. M.; Ahmad, N. D.; Moura, M. S. B. de; Bos, M. G. Analysis of energy fluxes and vegetation-atmosphere parameters in irrigated and natural ecosystems of semi-arid Brazil. Journal of Hydrology, v.362, p.110-127, 2008.

Wiedenfeld, B. Scheduling water application on drip irrigated sugarcane. Agricultural Water Management, v.64, p.169-181, 2004. 